\title{
A major gene for host response to porcine reproductive and respiratory syndrome is not unfavorably associated with overall performance under nonchallenging conditions in commercial pig lines ${ }^{1}$
}

\author{
J. R. Dunkelberger, ${ }^{* 2}$ P. K. Mathur, $\uparrow$ M. S. Lopes, $\uparrow \dagger$ E. F. Knol, $\uparrow$ and J. C. M. Dekkers* \\ *Department of Animal Science, Iowa State University, Ames 50011; †Topigs Norsvin Research Center, \\ Beuningen, 6640 AA, the Netherlands; and $\$$ Topigs Norsvin, Curitiba, 80420-210, Brazil
}

\begin{abstract}
A QTL for host response to porcine reproductive and respiratory syndrome (PRRS) was identified in a previous study. The SNP WUR10000125 (WUR) is used as a tag SNP for this QTL. The favorable (B) allele at this SNP is in low frequency in commercial populations, possibly because this allele is unfavorably associated with an important trait under nonchallenging conditions and, therefore, may have been selected against. Therefore, objectives of this study were to estimate the effect of WUR on traits under selection in commercial lines under nonchallenging conditions and to estimate the effect of WUR genotype of parents on performance of crossbred progeny in a commercial-like environment. Data were collected on 4 purebred lines: a Landrace dam line (D1), a Large White dam line (D2), a synthetic sire line (S1), and a Pietrain sire line (S2). Traits analyzed included total number born, number stillborn, farrowing survival, lactation survival, litter mortality, daily feed intake (DFI), backfat, average test daily gain (TGR), average lifetime daily gain (LGR), and Topigs Norsvin selection index (TSI) value, indicative of overall economic value. Deregressed EBV were calculated for each trait (except TSI) and
\end{abstract}

analyzed within line. In the $\mathrm{S} 1$ line, $\mathrm{AB}$ and $\mathrm{BB}$ pigs had significantly lower TGR $(P=0.002)$ and LGR $(P$ $=0.001)$ than AA pigs but also lower DFI $(P=0.004)$. Conversely, $\mathrm{AB}$ and $\mathrm{BB}$ pigs had significantly higher DFI $(P<0.001)$ and AB pigs had significantly higher TGR $(P=0.03)$ than AA pigs in the S2 line. The effect of WUR on TSI was not significant for any line $(P \geq 0.15)$. Analyses of phenotypic records collected on crossbred progeny of S1 sires and D1 $\times \mathrm{D} 2 \mathrm{~F}_{1}$ females showed no significant effect of parent WUR genotype on DFI, backfat, TGR, or LGR $(P \geq 0.07)$. In conclusion, the effect of WUR was nonsignificant for most traits but the magnitude and direction of the effect differed by trait and by line. The favorable allele for host response to PRRS was associated with greater DFI and a tendency for greater TGR in the S2 line, but the opposite direction of effect was detected for the S1 line. Regardless of the effect on individual traits, no significant effect of WUR on TSI was detected for any line. Therefore, selecting for the B allele is expected to result in progeny with increased resistance to PRRS without compromising overall economic value under normal, nonchallenging conditions.

Key words: disease, porcine reproductive and respiratory syndrome virus, swine, WUR10000125

\section{INTRODUCTION}

Previously, a major QTL for host response to porcine reproductive and respiratory syndrome

\footnotetext{
${ }^{1}$ This project was funded by the USDA NIFA grant 2012-3842019286 (National Needs Training Grant) and by Topigs Norsvin.

${ }^{2}$ Corresponding author: jenelled@iastate.edu

Received February 28, 2017.

Accepted May 11, 2017.
} (PRRS) was identified on chromosome 4 (Boddicker et al., 2012). Single nucleotide polymorphism WUR10000125 (WUR) is in complete linkage disequilibrium with the causative mutation and can 
be used as a tag SNP for this QTL (Boddicker et al., 2014a,b; Koltes et al., 2015). Guanylate binding protein 5 (GBP5), which plays a role in the innate immune response (Shenoy et al., 2012), was recently identified as the causative gene for this QTL (Koltes et al., 2015). The effect of WUR has been validated across breeds and genetic sources (Boddicker et al., 2014b) and following infection with 2 North American PRRS virus (PRRSV) isolates (Hess et al., 2016), vaccination with a PRRS modified live virus (MLV) vaccine (Abella et al., 2016; Dunkelberger et al., 2017), and coinfection with PRRSV and porcine circovirus type 2b (PCV2b; Dunkelberger et al., 2017).

The frequency of the favorable (B) allele ("G" nucleotide) under PRRS challenge is low in commercial populations (i.e., average frequency across 8 PRRS Host Genetics Consortium trials was 0.14 , where each trial comprised pigs sourced from different breeding companies; Boddicker et al., 2014b). One hypothesis for the low frequency of the B allele is that it is unfavorably associated with an important trait under nonchallenging conditions and, therefore, has been selected against in commercial breeding schemes.

Until now, information regarding the effect of WUR on performance under nonchallenging conditions has been limited, except for results from a recent study in which AB pigs had significantly slower growth than AA pigs under nonchallenging conditions (Abella et al., 2016). Therefore, the objectives of this study were to estimate the effect of WUR on traits under selection in commercial lines under nonchallenging conditions and to estimate the effect of parent WUR genotype on performance of crossbred progeny in a commercial-like environment.

\section{MATERIALS AND METHODS}

The data used for this study were obtained as part of routine data recording in a commercial breeding program. Samples collected for DNA extraction were used only for the routine diagnostic purpose of the breeding program. Data recording and sample collection were conducted strictly in line with the rules given by the Dutch law on the protection of animals (Gezondheids- en welzijnswet voor dieren).

\section{Animals and Genotypes}

Data used for this study were from 2 purebred dam lines (a Landrace dam line [D1] and a Large White dam line [D2]) and 2 purebred sire lines (a synthetic sire line [S1] and a Pietrain sire line [S2]) from Topigs Norsvin (Vught, the Netherlands) high-health nucleus farms. The number of individuals, sires, and dams per line used for analyses is presented in Table 1. Records
Table 1. Number of individuals, sires, and dams per line used for analyses

\begin{tabular}{lrcc}
\hline \hline Line $^{1}$ & No. & Number of sires & Number of dams \\
\hline D1 & 9,264 & 628 & 2,946 \\
D2 & 18,458 & 710 & 4,330 \\
S1 & 7,228 & 497 & 2,175 \\
S2 & 8,868 & 449 & 2,353 \\
\hline
\end{tabular}

${ }^{1} \mathrm{D} 1$ = Landrace dam line; D2 = Large White dam line; $\mathrm{S} 1$ = synthetic sire line; $\mathrm{S} 2=$ Pietrain sire line.

were collected on selection candidates from 40, 34, 8, and 19 farms for the D1, D2, S1, and S2 lines, respectively, from 2015 through 2016. Some farms vaccinated for PRRS using a type 1 PRRS MLV vaccine. For these farms, some pigs were vaccinated at weaning (i.e., $3 \mathrm{wk}$ of age), all pigs were vaccinated at $26 \mathrm{wk}$ of age, dams were vaccinated at d 60 of gestation, and dams received a booster vaccine at $6 \mathrm{~d}$ after farrowing.

Data were also collected on S1 $\times(\mathrm{D} 1 \times \mathrm{D} 2)$ crossbred finishing pigs of $\mathrm{S} 1$ sires and $\mathrm{D} 1 \times \mathrm{D} 2 \mathrm{~F}_{1}$ females to estimate the effect of parent WUR genotype on performance of crossbred progeny. Phenotypic records collected on 2,184 S1 $\times(\mathrm{D} 1 \times \mathrm{D} 2)$ individuals from 2 combined crossbred and purebred selection (Wei and van der Werf, 1994) farms, which mimic commercial conditions, were used for analyses. Pigs were from 148 purebred S1 sires and 316 D1 $\times$ D2 dams. Combined crossbred and purebred selection farms had conventional health status, meaning that any of the following pathogens could have been present: Actinobacillus pleuropneumoniae, Mycoplasma, Bordetella, nontoxigenic Pasteurella multocida, Streptococcus suis, Staphylococcus hyicus, Haemophilus parasuis, Escherichia coli, Clostridium perfringens, coccidiosis, Lawsonia, Salmonella spp., influenza, porcine circovirus, porcine respiratory corona virus, or PRRS. Farms were free of mange, Brachyspira, and atrophic rhinitis. Pigs were fed a conventional, commercial grow-to-finish ration ad libitum.

Per routine breeding program procedure, all selection candidates were genotyped using the PorcineSNP60 BeadChip (Illumina Inc., San Diego, CA), GeneSeek Custom 80K SNP chip (GeneSeek, Inc., Lincoln, NE), or the GeneSeek Custom 10K SNP chip (GeneSeek, Inc.). Crossbred progeny were genotyped using the GeneSeek Custom 10K SNP chip (GeneSeek, Inc.). Genotypes for the WUR SNP were extracted, and a $\chi^{2}$ test was performed to determine whether the observed genotype frequencies significantly differed from the expected genotype frequencies under Hardy-Weinberg equilibrium (HWE). At the WUR SNP, the B allele corresponds to the "G" nucleotide and is favorable under PRRSV infection. The B allele has shown to be 
dominant to the A allele, which corresponds to the "A" nucleotide (Boddicker et al., 2014b).

\section{Traits}

The 5 reproduction traits and 4 finishing traits that receive the greatest emphasis in the Topigs Norsvin selection index (TSI) were analyzed. The TSI combines EBV and economic values of traits according to the specific breeding goals of each line. Reproduction traits recorded at the sow level included total number born, defined as the number of live born piglets plus the number of stillborn piglets within a litter; number of stillborn piglets within a litter; and litter mortality, recorded as the number of piglets that died between birth and weaning. Reproduction traits recorded at the individual piglet level included farrowing survival (FSL; whether a piglet did [=1]/did not [=0] survive farrowing) and lactation survival (whether a live-born piglet $\mathrm{did}[=1] /$ did not $[=0]$ survive until weaning). Although recorded as binary traits, FSL and lactation survival were analyzed as continuous traits as the probabilities of survival. Finishing traits included daily feed intake (DFI), recorded in grams per day from the start to the end of the test period; backfat (BFE), recorded as fat depth at the end of the test period (approximately $120 \mathrm{~kg}$ ) in millimeters; average test daily gain (TGR), expressed in grams of weight gain per day from the start to the end of the test period; and average lifetime daily gain (LGR), expressed in grams of weight gain per day from birth to the end of the test period.

\section{Statistical Analyses}

Estimated Breeding Values and Deregressed $\boldsymbol{E B} \boldsymbol{V}$. Estimated breeding values for all evaluated traits were obtained from routine genetic evaluation of Topigs Norsvin lines using a multitrait model with MiXBLUP software (Mulder et al., 2012). Deregressed EBV (dEBV) were obtained for all individuals for all evaluated traits when the reliability of the $\operatorname{EBV}\left(r^{2}\right)$ was greater than $20 \%$ using the method described by Garrick et al. (2009). Parent average effects were also removed as part of the deregression process to obtain more accurate estimates of the Mendelian sampling component of each individual. Reliabilities $\left(r^{2}\right)$ were extracted from the genetic evaluation and were based on the methodology of Tier and Meyer (2004). Weighting factors based on the estimated reliability of dEBV were estimated following Garrick et al. (2009) using a value of 0.5 for the scalar c.

Analyses of the Effect of WUR10000125 on Index Traits. The effect of WUR on each evaluated trait was analyzed using dEBV for each trait as the response variable using a single-trait animal model in ASReml
4.0 (Gilmour et al., 2015). Each trait was analyzed by line using the following model:

$$
\begin{aligned}
\mathrm{Y}_{\mathrm{ijk}} \times \mathrm{wt}_{\mathrm{ijk}}= & \mu+\text { PRRS_Vacc }_{\mathrm{i}}+\mathrm{WUR}_{\mathrm{j}} \\
& + \text { PRRS_Vacc }_{\mathrm{i}} \times \mathrm{WUR}_{\mathrm{j}} \\
& + \text { Animal }_{\mathrm{k}}+\mathrm{e}_{\mathrm{ijk}}
\end{aligned}
$$

in which $\mathrm{Y}_{\mathrm{ijk}}=$ the observed $\mathrm{dEBV}, \mathrm{wt}_{\mathrm{ijk}}=$ weighting factor for the residual, $\mu=$ the overall mean, PRRS Vacc $_{i}=$ fixed class effect for PRRS vaccination status of farm (whether the farm that the pig originated from vaccinates [vaccinated $\{\mathbf{V} \mathbf{x}\}$ ], or not [nonvaccinated $\{\mathbf{N o n V \mathbf { x }}\}$ ], for PRRS), $\mathrm{WUR}_{\mathrm{j}}=$ fixed class effect of WUR genotype (AA, AB, or BB), PRRS_Vacc $\times \mathrm{WUR}_{\mathrm{j}}=$ interaction of PRRS vaccination status by WUR genotype, Animal $_{\mathrm{k}}=$ random animal genetic effect, and $\mathrm{e}_{\mathrm{ijk}}=$ the random residual effect. The interaction of PRRS vaccination status (PRRS_Vacc) $\times$ WUR was removed from the model when nonsignificant $(P \geq 0.10)$ and the analysis was rerun by replacing WUR with the terms WUR_add and WUR_dom, which represent contrasts fitted to obtain separate additive and dominance effects for WUR, respectively. When PRRS_Vacc $\times$ WUR was significant, the analysis was also rerun by replacing PRRS_Vacc $\times$ WUR with PRRS_Vacc $\times$ WUR_add and PRRS_Vacc $\times$ WUR_dom. However, because dEBV are based on the additive genetic effect, little to no effect of WUR_dom or PRRS_Vacc $\times$ WUR_dom was expected for these analyses.

The same model was used to estimate the effect of WUR on dEBV for each finishing trait for an analysis across lines with the following terms fitted as additional fixed effects: line (D1, D2, S1, or S2), line $\times$ PRRS_Vacc, and line $\times$ WUR. The latter interaction terms were removed from the model when nonsignificant $(P \geq 0.10)$.

Animal genetic effects were assumed distributed $\sim N\left(0, \mathbf{A} \sigma^{2}{ }_{\mathrm{a}}\right)$, in which $\mathbf{A}$ is the average numerator relationship matrix between individuals and $\sigma^{2}$ is the additive genetic variance. Genetic relationships were derived using a minimum of 13 generations of pedigree for each line. Residuals $\mathrm{e}_{\mathrm{ijk}}$ were assumed distributed $\sim N\left(0, \mathbf{D} \sigma^{2}{ }_{\mathrm{e}}\right)$, in which $\sigma^{2}{ }_{\mathrm{e}} \mathrm{ij}$ is the residual variance and $\mathbf{D}$ is a diagonal matrix calculated as $\mathbf{I} \times \mathbf{w t}$, in which $\mathbf{I}$ is an identity matrix and wt is a vector of weighting factors of the dEBV. The same models described above were also used to estimate the effect of WUR on TSI for each line separately or across lines. However, because actual values, rather than dEBV, were analyzed for TSI, no weighting factor was included.

A second objective was to estimate the effect of parent WUR genotype on performance of crossbred progeny. The following model was used to analyze 
TGR and LGR, recorded as grams of weight gain per day from the beginning of the test period or from birth to the end of the finishing period, respectively, using ASReml 4.0 (Gilmour et al., 2015), which is similar to that used for routine breeding value estimation:

$$
\begin{aligned}
\mathrm{Y}_{\mathrm{ijklm}}= & \mu+\text { Trial }_{\mathrm{j}}+\mathrm{FLS}_{\mathrm{k}}+\mathrm{FC}_{1}+\mathrm{B}_{1} \\
& \times \mathrm{BWS}_{\mathrm{i}}+\mathrm{B}_{2} \times \mathrm{Avg}_{\mathrm{i}}+\text { Animal }_{\mathrm{i}} \\
& + \text { Litter }_{\mathrm{m}}+\mathrm{e}_{\mathrm{ijklm}}
\end{aligned}
$$

in which $Y_{\mathrm{ijklm}}=$ the observed phenotype recorded on $\mathrm{S} 1 \times(\mathrm{D} 1 \times \mathrm{D} 2)$ progeny, $\mu=$ the overall mean, Trial $_{\mathrm{j}}=$ fixed class effect of trial (to account for differences in the feed formulation used), $\mathrm{FLS}_{\mathrm{k}}=$ fixed class effect of farm by line and sex, $\mathrm{FC}_{1}=$ fixed class effect of farm compartment (i.e., group of pens within a farm), $\mathrm{B}_{1} \times$ $\mathrm{BWS}_{\mathrm{i}}=$ partial regression on scaled birth weight (birth weight - average birth weight), $B_{2} \times \operatorname{Avg}_{i}=$ partial regression on the average WUR genotype of parents (the average number of copies of the $\mathrm{B}$ allele of the

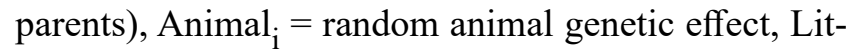
ter $_{\mathrm{m}}=$ random effect of litter, and $\mathrm{e}_{\mathrm{ijklm}}=$ the random residual effect. Animal genetic effects were assumed distributed $\sim N\left(0, \mathbf{A} \sigma^{2}{ }_{\mathrm{a}}\right)$, in which $\mathbf{A}$ is the numerator relationship matrix between individuals and $\sigma^{2}$ is the additive genetic variance. Litter effects were assumed distributed $\sim N\left(0, \mathbf{I} \sigma^{2}{ }_{\mathrm{L}}\right)$, in which $\mathbf{I}$ is the identity matrix and $\sigma_{L}^{2}$ is the unknown litter variance. Residuals $\mathrm{e}_{\mathrm{ijklm}}$ were assumed distributed $\sim N\left(0, \mathbf{I} \sigma^{2}{ }_{\mathrm{e}}\right)$, in which $\sigma^{2}$ is the residual variance.

Model [2] was also used to analyze BFE and DFI, recorded as fat depth at the end of the finishing period and feed intake from the start to the end of the finishing period, respectively. For analysis of BFE, the effects $\mathrm{B}_{1} \times \mathrm{HCW}_{\mathrm{i}} \times \operatorname{Group}_{\mathrm{k}}$ (partial regression on $\mathrm{HCW}$ ) and $\mathrm{B}_{2} \times \mathrm{HCW}_{\mathrm{i}}^{2} \times$ Group $_{\mathrm{k}}$ (partial regression on $\mathrm{HCW}$ squared) were fitted in place of $\mathrm{B}_{1} \times \mathrm{BWS}_{\mathrm{i}}$. For DFI, $\mathrm{B}_{1} \times$ Start Weight $_{\mathrm{i}}$ (partial regression on age at start of the finishing period) was fitted instead of $B_{1} \times B_{W}$.

\section{RESULTS}

The number of individuals by line and WUR genotype is presented in Table 2. The frequency of the B allele for WUR was $0.20,0.10,0.22$, and 0.18 for the D1, D2, S1, and S2 lines, respectively. Results of a $\chi^{2}$ test indicated that the observed genotype frequencies significantly differed from expected for the D2 line $(P$ $=0.0008)$ and the S2 line $(P<0.0001)$. For both lines, fewer heterozygotes and more BB pigs were observed than expected.
Table 2. Genotype frequencies for WUR10000125 by line

\begin{tabular}{lccccc}
\hline \hline & & \multicolumn{3}{c}{ Genotype frequency } & \\
\cline { 3 - 5 } Line $^{1}$ & MAF $^{2}$ & $\mathrm{AA}$ & $\mathrm{AB}$ & $\mathrm{BB}$ & $P$-value \\
\hline $\mathrm{D} 1$ & 0.20 & 0.64 & 0.32 & 0.04 & 0.20 \\
$\mathrm{D} 2$ & 0.10 & 0.81 & 0.18 & 0.01 & 0.0008 \\
$\mathrm{~S} 1$ & 0.22 & 0.61 & 0.34 & 0.05 & 0.26 \\
$\mathrm{~S} 2$ & 0.18 & 0.68 & 0.28 & 0.04 & $<0.0001$ \\
\hline
\end{tabular}

${ }^{1} \mathrm{D} 1$ = Landrace dam line; D2 = Large White dam line; $\mathrm{S} 1$ = synthetic sire line; $\mathrm{S} 2=$ Pietrain sire line.

${ }^{2} \mathrm{MAF}=$ minor allele frequency: frequency of the B allele.

${ }^{3} P$-value for a $\chi 2$ test of the observed versus expected genotype frequencies.

\section{Analyses of Purebred Data}

The $P$-values and least squares means for the effect of WUR on each evaluated trait by line are presented in Tables 3 and 4 for analyses of reproduction and finishing traits, respectively. The results indicate that the effect of WUR was significant for analyses of some traits but that the magnitude and direction of the effect differed by trait and by line. Because analyses of purebred lines were performed using dEBV for each trait, dominance effects were expected to be minimal and, therefore, only differences between the AA and AB genotypes will be emphasized. At the WUR SNP, the $\mathrm{B}$ allele has also been shown to act in a completely dominant manner to the A allele (Boddicker et al., 2014b).

Reproduction Traits. No significant effect of PRRS_Vacc $\times$ WUR or WUR was detected for analyses of reproduction traits for the D1 line $(P>0.20)$ or the D2 line $(P \geq 0.05)$. However, a tendency $(P=0.05)$ for a significant effect of PRRS_Vacc $\times$ WUR was detected for analysis of FSL for the D2 line (Table 3). No difference $(P>0.34)$ in FSL was detected between WUR genotypes within the NonVx group, but AB pigs had significantly $(P<0.001)$ greater FSL than AA pigs within the Vx group. Within the Vx group, the effect size for $\mathrm{AB}$ pigs $(0.18$, expressed in genetic $\mathrm{SD}$ units for the trait) was higher than for AA pigs.

Finishing Traits. No significant $(P \geq 0.33)$ effect of WUR on dEBV was detected for any of the finishing traits for the across-line analyses (Table 4). However, a significant $(P=0.004)$ effect of WUR $\times$ line was detected for TGR, driven by a significant $(P$ $=0.002)$ effect of WUR on TGR for the S1 line and a tendency for a significant $(P=0.09)$ effect of WUR on TGR for the S2 line. No significant $(P \geq 0.19)$ effect of WUR on TGR was detected for either of the dam lines. A significant $(P=0.004)$ effect of WUR $\times$ line was also detected for LGR, where a significant $(P=$ 0.002 ) effect of WUR on LGR was detected for the S1 line but not the $\mathrm{S} 2$, D1, or D2 lines $(P \geq 0.26)$. 
Table 3. The $P$-values and least squares means (SE) for the effect of WUR10000125 (WUR) on deregressed EBV by genotype and line for reproduction traits

\begin{tabular}{|c|c|c|c|c|c|c|c|c|c|}
\hline \multirow[b]{2}{*}{ Trait } & \multirow[b]{2}{*}{ Line $^{1}$} & \multicolumn{3}{|c|}{ Least squares mean } & \multirow[b]{2}{*}{$P$-value } & \multirow{2}{*}{$\begin{array}{l}\text { Additive } \\
\text { effect }^{2}\end{array}$} & \multirow{2}{*}{$\begin{array}{l}\text { Additive } \\
P \text {-value }\end{array}$} & \multirow{2}{*}{$\begin{array}{l}\text { Dominance } \\
\text { effect }^{3}\end{array}$} & \multirow{2}{*}{$\begin{array}{c}\text { Dominance } \\
P \text {-value }\end{array}$} \\
\hline & & AA & $\mathrm{AB}$ & $\mathrm{BB}$ & & & & & \\
\hline \multirow[t]{2}{*}{$\mathrm{TNB}^{4}$} & D1 & $-0.23^{\mathrm{a}}(0.08)$ & $-0.23^{\mathrm{a}}(0.09)$ & $-0.44^{\mathrm{a}}(0.22)$ & 0.61 & $-0.11(0.11)$ & 0.32 & $0.10(0.13)$ & 0.42 \\
\hline & $\mathrm{D} 2$ & $-0.23^{\mathrm{a}}(0.07)$ & $-0.34^{\mathrm{a}}(0.10)$ & $-0.42^{\mathrm{a}}(0.27)$ & 0.37 & $-0.10(0.13)$ & 0.47 & $-0.02(0.15)$ & 0.91 \\
\hline \multirow[t]{2}{*}{$\mathrm{STB}^{5}$} & D1 & $-0.11^{\mathrm{a}}(0.03)$ & $-0.06^{\mathrm{a}}(0.03)$ & $-0.04^{\mathrm{a}}(0.08)$ & 0.20 & $0.03(0.04)$ & 0.40 & $0.02(0.05)$ & 0.68 \\
\hline & D2 & $-0.12^{\mathrm{a}}(0.03)$ & $-0.06^{\mathrm{a}}(0.04)$ & $0.003^{\mathrm{a}}(0.11)$ & 0.14 & $0.06(0.05)$ & 0.24 & $-0.003(0.06)$ & 0.96 \\
\hline \multirow[t]{3}{*}{ FSL $^{6}$} & D1 & $1.67^{\mathrm{a}}(0.28)$ & $1.68^{\mathrm{a}}(0.30)$ & $1.61^{\mathrm{a}}(0.52)$ & 0.99 & $-0.03(0.24)$ & 0.90 & $0.04(0.27)$ & 0.88 \\
\hline & D2, $\operatorname{NonV}^{7}$ & $1.37^{\mathrm{a}}(0.38)$ & $1.49^{\mathrm{a}}(0.42)$ & $1.03^{\mathrm{a}}(0.67)$ & 0.63 & $-0.17(0.28)$ & 0.55 & $0.29(0.31)$ & 0.35 \\
\hline & $\mathrm{D} 2, \mathrm{Vx}^{8}$ & $1.30^{\mathrm{b}}(0.24)$ & $1.96^{\mathrm{a}}(0.29)$ & $2.82^{\mathrm{a}}(0.77)$ & $<0.001$ & $0.76(0.37)$ & 0.04 & $-0.10(0.39)$ & 0.79 \\
\hline \multirow[t]{2}{*}{ LSL $^{9}$} & D1 & $2.40^{\mathrm{a}}(0.39)$ & $2.14^{\mathrm{a}}(0.42)$ & $2.13^{\mathrm{a}}(0.73)$ & 0.62 & $-0.14(0.34)$ & 0.68 & $-0.13(0.39)$ & 0.73 \\
\hline & $\mathrm{D} 2$ & $2.90^{\mathrm{a}}(0.40)$ & $2.77^{\mathrm{a}}(0.45)$ & $1.40^{\mathrm{a}}(0.88)$ & 0.17 & $-0.75(0.40)$ & 0.06 & $0.62(0.44)$ & 0.16 \\
\hline \multirow[t]{2}{*}{$\mathrm{LMO}^{10}$} & D1 & $-0.44^{\mathrm{a}}(0.18)$ & $-0.57^{\mathrm{a}}(0.25)$ & $-0.95^{\mathrm{a}}(0.70)$ & 0.72 & $-0.26(0.36)$ & 0.47 & $0.13(0.43)$ & 0.76 \\
\hline & $\mathrm{D} 2$ & $-0.50^{\mathrm{a}}(0.24)$ & $-0.86^{\mathrm{a}}(0.32)$ & $-0.88^{\mathrm{a}}(0.84)$ & 0.37 & $-0.19(0.41)$ & 0.64 & $-0.17(0.47)$ & 0.70 \\
\hline
\end{tabular}

a,b Estimates within a row with different superscripts differ $(P<0.05)$.

${ }^{1} \mathrm{D} 1$ = Landrace dam line; D2 = Large White dam line.

${ }^{2}$ Additive effect of WUR.

${ }^{3}$ Dominance effect of WUR.

${ }^{4} \mathrm{TNB}=$ total number born: defined as the number of live born piglets plus the number of stillborn piglets within a litter.

${ }^{5} \mathrm{STB}=$ number stillborn: defined as the number of stillborn piglets within a litter.

${ }^{6} \mathrm{FSL}=$ farrowing survival: whether a piglet did $(=1) /$ did not $(=0)$ survive farrowing, analyzed as a continuous trait as the probability of surviving farrowing. There was suggestive evidence $(P=0.05)$ of a significant effect of WUR $\times$ porcine reproductive and respiratory syndrome (PRRS) vaccination status (PRRS_Vacc). $P$-values for the effect of PRRS_Vacc $\times$ contrasts fitted to obtain the additive effect of WUR (WUR_add) and PRRS_Vacc $\times$ contrasts fitted to obtain the dominance effect of WUR (WUR_dom) were $P=0.16$ and $P=0.02$, respectively.

${ }^{7} \mathrm{NonVx}=$ nonvaccinated: pigs were not vaccinated for PRRS.

${ }^{8} \mathrm{Vx}=$ vaccinated: pigs were vaccinated for PRRS.

${ }^{9} \mathrm{LSL}=$ lactation survival: whether a piglet $\operatorname{did}(=1) / \operatorname{did}$ not $(=0)$ survive until weaning, analyzed as a continuous trait as the probability of surviving until weaning.

${ }^{10} \mathrm{LMO}=$ litter mortality: recorded as the number of piglets that died between birth and weaning.

When each dam line was analyzed separately, no significant effect of PRRS_Vacc $\times$ WUR or WUR was detected for analyses of finishing traits $(P>0.15)$. However, a tendency $(P=0.07)$ for a significant effect of PRRS Vacc $\times$ WUR was detected for TGR for the D2 line (Table 4), where Vx AB pigs had significantly $(P=0.003 ; 0.39$ genetic SD) lower TGR than NonVx $\mathrm{AB}$ pigs and NonVx AA pigs and Vx AA pigs had significantly $(P=0.04$ and $P=0.02$, respectively; 0.10 and 0.32 genetic SD, respectively) lower TGR than NonVx AB pigs.

Several significant associations of WUR with finishing traits were detected for analysis of each sire line separately (Table 4). For the S2 line, AB pigs had significantly $(P=0.0006 ; 0.17$ genetic SD) greater DFI than AA pigs, but no difference in DFI was detected between $\mathrm{BB}$ and $\mathrm{AB}$ pigs $(P=0.13)$. Consequently, AB pigs also had significantly $(P=0.03 ; 0.09$ genetic SD) greater TGR than AA pigs, but no difference in TGR was detected between AA and BB $(P=0.20)$ or $\mathrm{AB}$ and $\mathrm{BB}(P=0.70)$ pigs.
The opposite direction of effect was detected for analyses of DFI and TGR for the S1 line, where AA pigs had significantly $(P=0.006 ; 0.14$ genetic SD) greater DFI than $\mathrm{AB}$ pigs and significantly $(P=0.006)$ greater DFI than BB pigs. However, AA pigs also had significantly $(P=0.01 ; 0.12$ genetic SD) greater TGR than $\mathrm{AB}$ pigs and significantly $(P=0.002)$ greater TGR than $\mathrm{BB}$ pigs. Results also indicate that AA pigs had significantly $(P=0.005 ; 0.13$ genetic $\mathrm{SD})$ greater LGR than $\mathrm{AB}$ pigs and significantly $(P=0.002)$ greater LGR than $\mathrm{BB}$ pigs.

Topigs Norsvin Selection Index. Results for the effect of WUR genotype on TSI within and across lines are presented in Table 5. No significant effect of WUR was detected on TSI for any one line $(P \geq 0.15)$ or across lines $(P=0.56)$, although numerically, $\mathrm{AB}$ pigs had the greatest TSI value for analyses of the dam lines and AA pigs had the greatest TSI value for analyses of the sire lines. 
Table 4. The $P$-values and least squares means (SE) for the effect of WUR10000125 (WUR) on deregressed EBV by genotype and line (or across lines) for finishing traits

\begin{tabular}{|c|c|c|c|c|c|c|c|c|c|}
\hline \multirow[b]{2}{*}{ Trait } & \multirow[b]{2}{*}{ Line $^{1}$} & \multicolumn{3}{|c|}{ Least squares mean } & \multirow[b]{2}{*}{$P$-value } & \multirow{2}{*}{$\begin{array}{l}\text { Additive } \\
\text { effect }^{2}\end{array}$} & \multirow{2}{*}{$\begin{array}{c}\text { Additive } \\
P \text {-value }\end{array}$} & \multirow{2}{*}{$\begin{array}{l}\text { Dominance } \\
\text { effect }^{3}\end{array}$} & \multirow{2}{*}{$\begin{array}{c}\text { Dominance } \\
P \text {-value }\end{array}$} \\
\hline & & AA & $\mathrm{AB}$ & $\mathrm{BB}$ & & & & & \\
\hline \multirow[t]{5}{*}{$\overline{\mathrm{BFE}^{4}}$} & D1 & $0.10^{\mathrm{a}}(0.15)$ & $0.15^{\mathrm{a}}(0.16)$ & $0.09^{\mathrm{a}}(0.18)$ & 0.38 & $-0.003(0.05)$ & 0.95 & $0.05(0.05)$ & 0.28 \\
\hline & D2 & $0.55^{\mathrm{a}}(0.18)$ & $0.63^{\mathrm{a}}(0.18)$ & $0.58^{\mathrm{a}}(0.21)$ & 0.15 & $0.01(0.06)$ & 0.83 & $0.06(0.06)$ & 0.31 \\
\hline & S1 & $0.39^{\mathrm{a}}(0.13)$ & $0.36^{\mathrm{a}}(0.13)$ & $0.33^{\mathrm{a}}(0.15)$ & 0.62 & $-0.03(0.04)$ & 0.45 & $-0.002(0.05)$ & 0.96 \\
\hline & S2 & $-0.11^{\mathrm{a}}(0.09)$ & $-0.13^{\mathrm{a}}(0.10)$ & $-0.15^{\mathrm{a}}(0.11)$ & 0.62 & $-0.02(0.03)$ & 0.55 & $-0.007(0.04)$ & 0.85 \\
\hline & Across lines & $0.24^{\mathrm{a}}(0.08)$ & $0.25^{\mathrm{a}}(0.08)$ & $0.21^{\mathrm{a}}(0.09)$ & 0.36 & $-0.01(0.02)$ & 0.62 & $0.03(0.03)$ & 0.21 \\
\hline \multirow[t]{5}{*}{$\mathrm{DFI}^{5}$} & D1 & $19.93^{\mathrm{a}}(13.43)$ & $30.76^{\mathrm{a}}(13.82)$ & $25.49^{\mathrm{a}}(19.97)$ & 0.24 & $2.78(8.02)$ & 0.72 & $8.05(8.93)$ & 0.37 \\
\hline & $\mathrm{D} 2$ & $31.91^{\mathrm{a}}(17.50)$ & $27.45^{\mathrm{a}}(18.34)$ & $34.18^{\mathrm{a}}(28.10)$ & 0.80 & $1.13(11.20)$ & 0.92 & $-5.60(12.01)$ & 0.64 \\
\hline & S1 & $22.94^{\mathrm{a}}(18.33)$ & $2.50^{\mathrm{b}}(18.73)$ & $-20.44^{b}(23.24)$ & 0.004 & $-21.69(7.94)$ & 0.01 & $1.25(8.95)$ & 0.88 \\
\hline & $\mathrm{S} 2$ & $15.57^{\mathrm{b}}(22.46)$ & $40.79^{\mathrm{a}}(23.32)$ & $65.01^{\mathrm{a}}(27.82)$ & $<0.001$ & $24.72(8.43)$ & 0.004 & $0.50(8.99)$ & 0.95 \\
\hline & Across lines & $22.23^{\mathrm{a}}(8.96)$ & $23.88^{\mathrm{a}}(9.28)$ & $22.50^{\mathrm{a}}(12.26)$ & 0.90 & $0.14(4.43)$ & 0.97 & $1.52(4.86)$ & 0.75 \\
\hline \multirow[t]{6}{*}{$\mathrm{TGR}^{6}$} & D1 & $7.40^{\mathrm{a}}(4.59)$ & $10.22^{\mathrm{a}}(4.67)$ & $8.15^{\mathrm{a}}(6.11)$ & 0.28 & $0.37(2.21)$ & 0.86 & $2.45(2.41)$ & 0.31 \\
\hline & $\mathrm{D} 2, \mathrm{NonVx}^{7}$ & $11.17^{\mathrm{a}}(6.72)$ & $16.42^{\mathrm{a}}(7.07)$ & $15.32^{\mathrm{a}}(9.93)$ & 0.19 & $2.08(3.76)$ & 0.58 & $3.17(4.10)$ & 0.44 \\
\hline & $\mathrm{D} 2, \mathrm{Vx}^{8}$ & $-0.51^{\mathrm{a}}(5.35)$ & $-4.59^{\mathrm{a}}(5.76)$ & $-7.61^{\mathrm{a}}(11.93)$ & 0.30 & $-3.55(5.41)$ & 0.51 & $-0.54(5.64)$ & 0.92 \\
\hline & S1 & $-3.49^{\mathrm{a}}(6.11)$ & $-9.66^{\mathrm{b}}(6.24)$ & $-19.16^{b}(7.66)$ & 0.002 & $-7.84(2.56)$ & 0.002 & $1.67(2.87)$ & 0.56 \\
\hline & $\mathrm{S} 2$ & $-6.83^{b}(7.64)$ & $-1.81^{\mathrm{a}}(7.91)$ & $0.17^{\mathrm{ab}}(9.34)$ & 0.09 & $3.50(2.75)$ & 0.21 & $1.52(2.91)$ & 0.60 \\
\hline & Across lines ${ }^{9}$ & $-0.91^{\mathrm{a}}(2.85)$ & $-0.24^{\mathrm{a}}(2.94)$ & $-2.35^{\mathrm{a}}(3.81)$ & 0.44 & $-0.72(1.33)$ & 0.49 & $1.39(1.45)$ & 0.21 \\
\hline \multirow[t]{5}{*}{$\mathrm{LGR}^{10}$} & D1 & $7.00^{\mathrm{a}}(2.74)$ & $8.38^{\mathrm{a}}(2.80)$ & $5.91^{\mathrm{a}}(3.74)$ & 0.36 & $-0.55(1.39)$ & 0.69 & $1.92(1.52)$ & 0.21 \\
\hline & $\mathrm{D} 2$ & $3.21^{\mathrm{a}}(3.24)$ & $2.24^{\mathrm{a}}(3.38)$ & $2.00^{\mathrm{a}}(4.99)$ & 0.74 & $-0.60(1.93)$ & 0.75 & $-0.36(2.07)$ & 0.86 \\
\hline & S1 & $-0.27^{\mathrm{a}}(3.94)$ & $-4.69^{b}(4.02)$ & $-10.89^{b}(4.96)$ & 0.001 & $-5.31(1.67)$ & 0.002 & $0.89(1.88)$ & 0.63 \\
\hline & S2 & $-3.87^{\mathrm{a}}(4.83)$ & $-1.46^{\mathrm{a}}(5.00)$ & $-1.07^{\mathrm{a}}(5.89)$ & 0.26 & $1.40(1.73)$ & 0.42 & $1.02(1.83)$ & 0.58 \\
\hline & Across lines ${ }^{11}$ & $1.66^{\mathrm{a}}(1.79)$ & $1.35^{\mathrm{a}}(1.85)$ & $-0.57^{\mathrm{a}}(2.41)$ & 0.33 & $-1.12(0.85)$ & 0.14 & $0.81(0.93)$ & 0.27 \\
\hline
\end{tabular}

${ }^{\mathrm{a}, \mathrm{b}}$ Estimates within a row with different superscripts differ $(P<0.05)$.

${ }^{1} \mathrm{D} 1$ = Landrace dam line; D2 = Large White dam line; S1 = synthetic sire line; S2 = Pietrain sire line.

${ }^{2}$ Additive effect of WUR.

${ }^{3}$ Dominance effect of WUR.

${ }^{4} \mathrm{BFE}=$ backfat: recorded as fat depth at the end of the test period (approximately $120 \mathrm{~kg}$ ) in millimeters.

${ }^{5} \mathrm{DFI}=$ daily feed intake: recorded in grams per day from start to end of the test period.

${ }^{6} \mathrm{TGR}=$ test daily gain: expressed in grams of weight gain per day from start to end of the test period. There was suggestive evidence $(P=0.07)$ of a significant effect of WUR $\times$ porcine reproductive and respiratory syndrome (PRRS) vaccination status (PRRS_Vacc). $P$-values for the effect of PRRS_Vacc $\times$ contrasts fitted to obtain the additive effect of WUR (WUR_add) and PRRS_Vacc $\times$ contrasts fitted to obtain the dominance effect of WUR (WUR_dom) were $P=0.85$ and $P=0.17$, respectively.

${ }^{7} \mathrm{NonVx}=$ nonvaccinated: pigs were not vaccinated for PRRS.

${ }^{8} \mathrm{Vx}=$ vaccinated: pigs were vaccinated for PRRS

${ }^{9}$ A significant effect of WUR $\times$ line $(P=0.004)$ was detected. $P$-values for the effect of WUR_add $\times$ line and WUR_dom $\times$ line were $P=0.01$ and $P=$ 0.96 , respectively.

${ }^{10} \mathrm{LGR}=$ lifetime daily gain: expressed in grams of weight gain per day from birth until end of the test period.

${ }^{11}$ A significant effect of WUR $\times$ line $(P=0.004)$ was detected. $P$-values for the effect of WUR_add $\times$ line and WUR_dom $\times$ line were $P=0.02$ and $P$ $=0.85$, respectively.

\section{Analyses of Crossbred Data}

Results of phenotypic analyses of the $\mathrm{S} 1 \times(\mathrm{D} 1 \times$ D2) crossbred progeny showed no significant effect of average parent WUR genotype on DFI, BFE, TGR, or LGR ( $P \geq 0.07$; Table 6). However, there was suggestive evidence $(P=0.07)$ for an effect of average parent WUR genotype on BFE, for which BFE of S1 $\times(\mathrm{D} 1 \times$ D2) progeny increased by $0.59 \pm 0.32 \mathrm{~mm}$ for every 1 unit increase in the average number of $\mathrm{B}$ alleles carried by parents at the WUR SNP.

\section{DISCUSSION}

This is the first study to estimate the effect of WUR genotype, a tag SNP for a major QTL for host response to PRRS, on both reproduction and finishing traits in commercial pig lines under nonchallenging conditions. Since the initial identification of the QTL, other studies have validated the effect of WUR across breeds and breeding companies (Boddicker et al., 2014a,b), on infection with 2 North American PRRSV isolates (Hess et al., 2016), following vaccination with a type 1 (Abella et al., 2016) and type 2 PRRS MLV vaccine 
Table 5. The $P$-values and least squares means (SE) for the effect of WUR10000125 (WUR) genotype on Topigs Norsvin selection index value by genotype and line or across lines

\begin{tabular}{|c|c|c|c|c|c|c|c|c|}
\hline \multirow[b]{2}{*}{ Line $^{1}$} & \multicolumn{3}{|c|}{ Least squares mean } & \multirow[b]{2}{*}{$P$-value } & \multirow{2}{*}{$\begin{array}{l}\text { Additive } \\
\text { effect }^{2}\end{array}$} & \multirow{2}{*}{$\begin{array}{c}\text { Additive } \\
P \text {-value }\end{array}$} & \multirow{2}{*}{$\begin{array}{c}\text { Dominance } \\
\text { effect }^{3}\end{array}$} & \multirow{2}{*}{$\begin{array}{c}\text { Dominance } \\
P \text {-value }\end{array}$} \\
\hline & AA & $\mathrm{AB}$ & $\mathrm{BB}$ & & & & & \\
\hline$\overline{\mathrm{D} 1}$ & $93.77^{\mathrm{a}}(1.75)$ & $93.87^{\mathrm{a}}(1.75)$ & $93.39^{\mathrm{a}}(1.86)$ & 0.74 & $-0.19(0.37)$ & 0.60 & $0.29(0.38)$ & 0.44 \\
\hline D2 & $82.40^{\mathrm{a}}(1.79)$ & $82.75^{\mathrm{a}}(1.80)$ & $81.72^{\mathrm{a}}(1.94)$ & 0.15 & $-0.34(0.39)$ & 0.38 & $0.69(0.38)$ & 0.07 \\
\hline S1 & $90.07^{\mathrm{a}}(1.49)$ & $89.76^{\mathrm{a}}(1.50)$ & $89.69^{\mathrm{a}}(1.57)$ & 0.48 & $-0.19(0.27)$ & 0.49 & $-0.12(0.27)$ & 0.65 \\
\hline S2 & $89.92^{\mathrm{a}}(1.46)$ & $89.78^{a}(1.48)$ & $89.36^{\mathrm{a}}(1.59)$ & 0.69 & $-0.28(0.33)$ & 0.39 & $0.13(0.32)$ & 0.67 \\
\hline Across lines ${ }^{4}$ & $89.34^{\mathrm{a}}(0.86)$ & $89.35^{\mathrm{a}}(0.86)$ & $89.02^{\mathrm{a}}(0.91)$ & 0.56 & $-0.16(0.17)$ & 0.35 & $0.17(0.17)$ & 0.32 \\
\hline
\end{tabular}

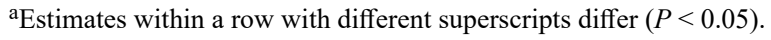

${ }^{1} \mathrm{D} 1$ = Landrace dam line; D2 = Large White dam line; $\mathrm{S} 1$ = synthetic sire line; $\mathrm{S} 2$ = Pietrain sire line.

${ }^{2}$ Additive effect of WUR.

${ }^{3}$ Dominance effect of WUR.

${ }^{4}$ A significant effect of line $\times$ porcine reproductive and respiratory syndrome vaccination status $(P<0.001)$ was detected.

(Dunkelberger et al., 2017), and on coinfection with PCV2b (Dunkelberger et al., 2017). However, prior to implementing selection for WUR to breed pigs for improved host response to PRRS, it must be established that the favorable (B) allele under PRRS challenge does not have a detrimental effect on an important selection index trait under nonchallenging conditions; hence, the motivation for this study.

Results of the $\chi^{2}$ test showed significant deviation from HWE for WUR for the D2 and the S2 lines. For both lines, deviations from HWE were driven by fewer heterozygotes and more BB pigs than expected. The most likely explanation for this result is that with such large sample sizes, even small deviations of the observed genotype count from the expected genotype count can produce a significant result. For example, the observed/expected frequencies of the BB genotype were $1.25 / 1.00 \%$ and $3.90 / 3.16 \%$, for the D2 and the S2 lines, respectively. Within line, differ-

Table 6. Estimate (SE) and $P$-value for the effect of average parent WUR10000125 (WUR) genotype for index traits of commercial crossbred progeny

\begin{tabular}{lcrlc}
\hline \hline Trait & Raw trait mean & \multicolumn{1}{c}{ SD } & \multicolumn{1}{c}{$\mathrm{ASE}^{1}$} & $P$-value \\
\hline $\mathrm{DFF}^{2}$ & $2,332.2$ & 255.9 & $9.80(28.93)$ & 0.73 \\
$\mathrm{BFE}^{3}$ & 13.9 & 2.7 & $0.59(0.32)$ & 0.07 \\
$\mathrm{TGR}^{4}$ & 894.6 & 86.7 & $3.90(11.61)$ & 0.73 \\
$\mathrm{LGR}^{5}$ & 552.0 & 48.0 & $2.59(6.03)$ & 0.66 \\
\hline
\end{tabular}

${ }^{1} \mathrm{ASE}=$ allele substitution effect: average WUR genotype of the parents where genotype was coded as the number of copies of the B allele.

${ }^{2} \mathrm{DFI}=$ daily feed intake: recorded in grams per day from start to end of the finishing period.

${ }^{3} \mathrm{BFE}=$ backfat: recorded as fat depth at the end of the finishing period in millimeters.

${ }^{4} \mathrm{TGR}=$ test daily gain: expressed in grams of weight gain per day from start to end of the finishing period.

${ }^{5} \mathrm{LGR}=$ lifetime daily gain: expressed in grams of weight gain per day from birth to end of the finishing period. ences between these observed and expected genotype frequencies were less than 0.75 . Therefore, although a significant departure from HWE was observed for WUR for these lines, these deviations are not of practical relevance.

One reason that the association of the $\mathrm{B}$ allele with reproduction and/or finishing traits under nonchallenging conditions was brought into question is because there is a low frequency of the $\mathrm{B}$ allele within commercial populations. One hypothesis for the observed low frequency of this allele is that the $\mathrm{B}$ allele may be unfavorably associated with an index trait under nonchallenging conditions and, therefore, may have been selected against in high-health nucleus facilities. Although some potentially unfavorable associations of WUR with index traits were detected for analyses of the sire lines in support of this hypothesis, the magnitude and direction of effect differed by trait and by line and, due to small effect sizes, are not considered to be of practical relevance.

The 5 reproduction traits and 4 finishing traits evaluated in this study were selected for analysis because they receive the greatest emphasis in the selection index for dam lines and sire lines, respectively. Results showed no significant effect of WUR on any of the reproduction or finishing traits in the dam lines, except FSL, for which a significant interaction of PRRS_Vacc $\times$ WUR was detected for the D2 line. For this line, no evidence of a difference in FSL was detected between genotypes within the NonVx group, but AB pigs had significantly greater FSL than AA pigs within the $\mathrm{Vx}$ group. Pigs in the latter group originated from farms that vaccinate dams with a PRRS MLV vaccine at $d 60$ of gestation. The PRRS MLV vaccine is a live virus vaccine, meaning that piglets may have been exposed to PRRSV during gestation. Therefore, this result indicates that of piglets potentially positive for PRRSV at 
farrowing, $\mathrm{AB}$ pigs had a significantly higher chance of surviving farrowing than AA pigs, which is consistent with previously reported results of a protective effect of the B allele following PRRS MLV vaccination (Dunkelberger et al., 2017).

Some significant associations of WUR with index traits were detected for analyses of the sire lines, but the magnitude and direction of this effect differed by trait and by line. For DFI, TGR, and LGR, the direction of the WUR effect was consistent within each line, but the opposite direction of effect was detected across lines. For instance, AB pigs had significantly lower DFI and, consequently, lower TGR and LGR than AA pigs for the $\mathrm{S} 1$ line, but the opposite direction of effect was detected for the S2 line. For the S2 line, AB pigs had significantly greater DFI and significantly greater TGR than AA pigs. However, regardless of the magnitude or direction of these effects for the S1 and the S2 lines, effect sizes of WUR for DFI, TGR, and LGR (expressed as a proportion of the genetic SD) were small. Therefore, although significant associations with WUR were detected for these traits, these associations are likely not of practical relevance.

Furthermore, although $\mathrm{AB}$ pigs had lower DFI, TGR, and LGR for the S1 line, overall impact on feed efficiency may not be negative, because the direction of the effect was the same for feed intake and growth. This was evaluated for the S1 line using the WUR genotype effects for the $\mathrm{S} 1$ line and the phenotypic means for DFI and TGR for the $\mathrm{S} 1 \times(\mathrm{D} 1 \times \mathrm{D} 2)$ individuals. The same G:F (i.e., g of weight gain/d during the test period to $\mathrm{g}$ of feed consumed/d during the test period) was observed for $\mathrm{AA}, \mathrm{AB}$, and $\mathrm{BB}$ pigs $(0.38: 1)$. The $\mathrm{G}: \mathrm{F}$ for $\mathrm{S} 1 \times(\mathrm{D} 1 \times \mathrm{D} 2)$ progeny was also $0.38: 1$ for $\mathrm{AA}, \mathrm{AB}$, and $\mathrm{BB}$ pigs. However, it is important to note that the SE of the WUR effect for BB pigs was large, due to a low number $(n=73)$ of BB S1 $\times(\mathrm{D} 1 \times \mathrm{D} 2)$ individuals. Therefore, although differences in the magnitude of the WUR effect were observed for feed intake and growth for the S1 line, these differences are likely not practically relevant and do not appear to negatively impact G:F of AB S1 or crossbred individuals, which might also explain why WUR had no significant effect on TSI for this line.

Taken together, few significant associations of WUR with index traits were detected for the purebred commercial lines used for this study. For associations that were detected, the magnitude and direction of effect differed by trait and by line. For the significant associations that were detected, the small effect sizes indicate little practical relevance of WUR genotype on the trait of interest. Results from analyses of each line separately and the across-line analyses indicate failure to identify a significant, negative effect of WUR across lines for any one trait. Results for the TSI analyses showed no significant effect of WUR on overall TSI for analysis of each line separately or analysis of TSI across lines. The results of the TSI analyses are particularly important because they indicate that the combined effect of WUR on all traits included in the selection index (whether favorable or unfavorable for individual traits) did not have a significant effect on the overall economic value of individuals within a line. This finding provides additional support for selection for the B allele to breed pigs for improved host response to PRRS.

Based on the finding that no consistent, negative association of WUR with any one trait was detected across lines, it remains unclear why the $\mathrm{B}$ allele is in low frequency in commercial populations. The negative association with growth rate for the S1 line may partially explain the low frequency of this allele in that line. However, the opposite direction of effect on growth rate was observed for the S2 line. This could be because the linkage phase between WUR and the causative mutation are opposite in these 2 lines. However, Koltes et al. (2015) showed that the putative causative mutation was perfectly concordant with WUR genotype for pigs with different haplotypes in the WUR region. Analyzing growth rate at different stages of production as separate traits may also provide some clarity regarding the effect of WUR on growth. Second, we cannot rule out that the low frequency of the $B$ allele may be due to a negative association of WUR with a trait that has been under (natural) selection and that was not analyzed in the current study. However, perhaps the most likely reason for the low frequency of the favorable allele at WUR is that there is no selective advantage of the B allele in the absence of disease and, therefore, this allele has not been selected for because most selection is performed in a clean environment.

Prior to this study, information regarding the effect of WUR under nonchallenging conditions was limited to the effect of WUR on reproductive performance in gestating sows (Serão et al., 2014) and growth rate in finishing pigs (Abella et al., 2016). In general, results from the current study are consistent with those reported by Serão et al. (2014), who showed that prior to a natural PRRS outbreak, the effect of WUR on reproductive performance of commercial gestating females was nonsignificant for all traits analyzed, except number weaned. For this trait, an unfavorable association of WUR with reproductive performance was detected, where the B allele was associated with significantly fewer piglets weaned (Serão et al., 2014).

For the effect of WUR on growth rate under nonchallenging conditions, results reported by Dunkelberger et al. (2017) showed that AB pigs grew significantly faster than AA pigs when vaccinated for PRRS 
but not significantly different from AA pigs when not vaccinated for PRRS. These results conflict with findings from a recent study where $\mathrm{AB}$ pigs had significantly slower growth than AA pigs during finishing (Abella et al., 2016). Possibly, this discrepancy can be attributed to the fact that pigs used for the Abella et al. (2016) study were $6 \mathrm{wk}$ older than those used for the study conducted by Dunkelberger et al. (2017). However, results reported by Abella et al. (2016) agree with results reported for the S1 line, where the favorable (B) allele under PRRSV infection was unfavorably associated with growth during finishing. Compared with the study conducted by Abella et al. (2016), for the current study, different breeds were used, growth rate was measured over a longer period of time, and at least 10 times the number of individuals per line was used for analyses.

The second objective of this study was to estimate the effect of average parent WUR genotype on performance of crossbred progeny. The $\mathrm{S} 1 \times(\mathrm{D} 1 \times \mathrm{D} 2)$ pigs that were used for this objective were the progeny of $\mathrm{S} 1$ sires and $\mathrm{D} 1 \times \mathrm{D} 2 \mathrm{~F}_{1}$ females. Daily feed intake, BFE, TGR, and LGR were analyzed using phenotypic records collected on $\mathrm{S} 1 \times(\mathrm{D} 1 \times \mathrm{D} 2)$ progeny in test stations. Results indicate no significant effect of average parent WUR genotype on performance for any of the traits. Therefore, WUR genotype of the parents did not negatively impact DFI, BFE, TGR, or LGR of their commercial crossbred offspring. Further analysis of WUR genotype of the $\mathrm{S} 1 \times(\mathrm{D} 1 \times \mathrm{D} 2)$ individuals themselves also showed no significant effect of their own WUR genotype on DFI, BFE, TGR, or LGR (data not shown). Because the farms where the crossbred pigs were raised were designed to mimic commercial conditions, the finding that neither parent average WUR genotype nor WUR genotype of the individual itself had a significant effect on any of the evaluated traits indicates that WUR genotype did not negatively impact performance of commercial crossbred finishing pigs reared in a commercial setting.

Based on these results, our recommendation to the industry is to sort boars for the B allele and to market these pigs as boars with naturally higher resistance to PRRS. This approach may be used as a simpler and less time-intensive alternative to including WUR genotype in the selection index. Instead, index value calculation and identification of top-ranking sires can be performed normally followed by selection of sires with the BB genotype. It is expected that progeny of $\mathrm{BB}$ sires, which will have at least one copy of the B allele, will have increased resistance to PRRS and no significant difference in TSI from progeny of AA or $\mathrm{AB}$ sires. Of the lines analyzed in this study, we recommend to begin sorting S1 sires. Although negative associations with WUR were detected for feed intake and growth rate for this line, no significant effect of WUR was detected on TSI, the frequency of the B allele is highest within the S1 line, and pigs from this line are already noted for increased robustness to disease challenge and environmental stressors in the field.

\section{Conclusions}

In conclusion, some significant associations of WUR with index traits were detected, but the magnitude and direction of the effect differed by trait and by line. With the exception of FSL, no effect of WUR was detected on reproduction or finishing traits for the dam lines under nonchallenging conditions. Significant associations of WUR with finishing traits were detected for the sire lines but with the opposite direction of effect across lines. The favorable (B) allele for host response to PRRS was associated with significantly higher feed intake and a tendency for faster growth in the S2 line but significantly lower feed intake and significantly slower growth in the S1 line.

However, despite significant effects detected on individual traits, these effects are not expected to be practically relevant and no significant effect of WUR on overall selection index value was detected for any line. Furthermore, neither parent WUR genotype nor WUR genotype of the individual itself had a significant effect on performance of $\mathrm{S} 1 \times(\mathrm{D} 1 \times \mathrm{D} 2)$ crossbred progeny reared in commercial-like conditions. Based on these results, selecting for the B allele is expected to result in progeny with increased resistance to PRRS without compromising overall economic value under normal, nonchallenging conditions.

\section{LITERATURE CITED}

Abella, G., R. N. Pena, C. Nogareda, R. Armengol, A. Vidal, L. Moradell, V. Tarancon, E. Novell, J. Estany, and L. Fraile. 2016. A WUR SNP is associated with European porcine reproductive and respiratory virus syndrome resistance and growth performance in pigs. Res. Vet. Sci. 104:117-122. doi:10.1016/j. rvsc.2015.12.014

Boddicker, N. J., A. Bjorkquist, R. R. R. Rowland, J. K. Lunney, J. M. Reecy, and J. C. M. Dekkers. 2014b. Genome-wide association and genomic prediction for host response to porcine reproductive and respiratory syndrome virus infection. Genet. Sel. Evol. 46(18):1-14. doi:10.1186/1297-9686-46-18

Boddicker, N. J., D. J. Garrick, R. R. R. Rowland, J. K. Lunney, J. M. Reecy, and J. C. M. Dekkers. 2014a. Validation and further characterization of a major quantitative trait locus associated with host response to experimental infection with porcine reproductive and respiratory syndrome virus. Anim. Genet. 45:4858. doi:10.1111/age.12079

Boddicker, N., E. H. Waide, R. R. R. Rowland, J. K. Lunney, D. J. Garrick, J. M. Reecy, and J. C. M. Dekkers. 2012. Evidence for a major QTL associated with host response to porcine reproductive and respiratory syndrome virus challenge. J. Anim. Sci. 90:1733-1746. doi:10.2527/jas.2011-4464 
Dunkelberger, J. R., N. V. L. Serão, M. C. Niederwerder, M. A. Kerrigan, J. K. Lunney, R. R. R. Rowland, and J. C. M. Dekkers. 2017. Effect of a major quantitative trait locus for porcine reproductive and respiratory syndrome (PRRS) resistance on response to coinfection with PRRS virus and porcine circovirus type $2 \mathrm{~b}$ (PCV2b) in commercial pigs, with or without prior vaccination for PRRS. J. Anim. Sci. 95:584-598. doi:10.2527/ jas.2016.1071

Garrick, D. J., J. F. Taylor, and R. L. Fernando. 2009. Deregressing estimated breeding values and weighting information for genomic regression analyses. Genet. Sel. Evol. 41(55):1-8. doi:10.1186/1297-9686-41-55

Gilmour, A. R., B. J. Gogel, B. R. Cullis, S. J. Welham, and R. Thompson. 2015. ASReml user guide release 4.1 structural specification. VSN International Ltd., Hemel Hempstead, UK.

Hess, A. S., Z. Islam, M. K. Hess, R. R. R. Rowland, J. K. Lunney, A. D. Wilson, G. S. Plastow, and J. C. M. Dekkers. 2016. Comparison of host genetic factors influencing pig response to infection with two North American isolates of porcine reproductive and respiratory syndrome virus. Genet. Sel. Evol. 48(43)1-20. doi:10.1186/s12711-016-0222-0
Koltes, J. E., E. Fritz-Waters, C. J. Eisley, I. Choi, H. Bao, A. Kommadath, N. V. L. Serão, N. J. Boddicker, S. M. Abrams, M. Schroyen, H. Loyd, C. K. Tuggle, G. S. Plastow, L. Guan, P. Stothard, J. K. Lunney, P. Liu, S. Carpenter, R. R. R. Rowland, J. C. M. Dekkers, and J. M. Reecy. 2015. Identification of a putative quantitative trait nucleotide in guanylate binding protein 5 for host response to PRRS virus infection. BMC Genomics 16(412):1-13. doi:10.1186/s12864-015-1635-9

Mulder, H., M. Lidauer, I. Stranden, E. Mantysaari, M. Pool, and R. Veerkamp. 2012. MiXBLUP manual. Animal Breeding and Genomics Center, Wageningen UR Livestock Research, Lelystad, the Netherlands.

Serão, N. V. L., O. Matika, R. A. Kemp, J. C. S. Harding, S. C. Bishop, G. S. Plastow, and J. C. M. Dekkers. 2014. Genetic analysis of reproductive traits and antibody response in a PRRS outbreak herd. J. Anim. Sci. 92:2905-2921. doi:10.2527/ jas.2014-7821

Shenoy, A. R., D. A. Wellington, P. Kumar, H. Kassa, C. J. Booth, P. Cresswell, and J. D. MacMicking. 2012. GBP5 promotes NLRP3 inflammasome assembly and immunity in mammals. Science 336:481-485. doi:10.1126/science.1217141

Tier, B. B., and K. Meyer. 2004. Approximating prediction error covariances among additive genetic effects within animals in multiple-trait and random regression models. J. Anim. Breed. Genet. 121:77-89. doi:10.1111/j.1439-0388.2003.00444.x

Wei, M., and J. H. J. van der Werf. 1994. Maximizing genetic response in crossbreds using both purebred and crossbred information. Anim. Sci. 59:401-413. 